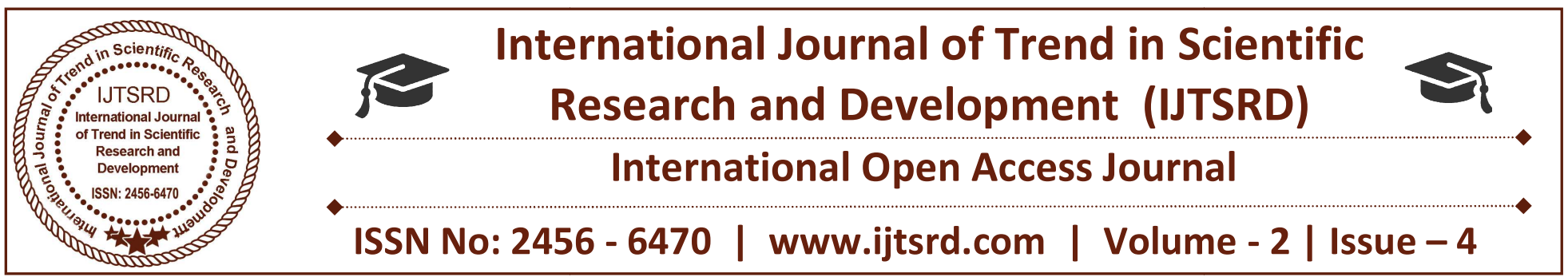

\title{
Cloud Computing Using Amazon Web Services (AWS)
}

\author{
Suyog Bankar \\ SIES College of Management Studies, (SIESCOMS), Nerul, \\ Navi Mumbai, Maharashtra, India
}

\section{ABSTRACT}

Cloud computing is becoming an increasingly popular enterprise model in which computing resources are made available on-demand to the user as needed. The unique value proposition of cloud computing creates new opportunities to align IT and business goals. Cloud computing use the internet technologies for delivery of IT-Enabled capabilities 'as a service' to any needed users i.e. through cloud computing we can access anything that we want from anywhere to any computer without worrying about anything like about their storage, cost, management and so on. In here we will study about 'Amazon Web Services (AWS)' which is one of the best cloud service provider on the world. AWS is the most trusted provider of cloud computing which not only provides the excellent cloud security but also provides excellent cloud services. In this paper I provide a comprehensive study on the motivation factors of adopting I. Introduction Cloud computing using AWS, review the several cloud deployment and service models of AWS. It also explore certain benefits of cloud computing using AWS over traditional IT service environment-including scalability, flexibility, reduced capital and higher resource utilization are considered as adoption reasons for cloud computing AWS environment. AWS is low cost, pay per use, highly secure, easy to use cloud service and many more.

Keywords: Amazon Web Services (AWS), Cloud Computing, Cloud Services, Scalability, AutoScaling.

\section{Scope}

The main objective of this document is to tell people why aws is more better technology while using cloud computing.aws is the market trending technology in IT cloud industry now days.so people should aware about benefits of cloud computing.

\section{Literature Survey}

The cloud computing technique has consist of many service providers but there was some problems while using cloud computing in IT industry. People were not sure about reliability of cloud computing few years ago because all data of companies are online on the cloud and can access can from anywhere and by anyone. There were some issues in cloud computing before introduce of aws in market which are listed below;-

The major problems in Cloud Computing technique are:

1. Data Security Concern

2. Selecting the perfect cloud setup

3. Real time monitoring requirement

4. Cost barrier

5. Unauthorized Service providers

The AWS is a technology which resolves all cloud computing related issues. It gives highly secure infrastructure. Using aws you need not to worry about maintaining data centers because here everything is manage by aws. Using aws you easily deploy your application in multiple regions around the world within minutes. Aws is very cost effective, highly scalable, more secure best cloud service providers in the world.

\section{Existing system}

The existing infrastructure of aws provides services like compute, storage, database,

Analytics, networking, mobile, developer tools, IoT, security and enterprises applications. These services helps Organizations to move faster, lower IT costs and Scale. Aws is trusted by the largest enterprises 
and the hottest start-ups to power wide variety of game development, Data processing and warehousing, storage, archive, and many others.

\section{Challenges in Cloud Computing}

1. Cloud computing itself is affordable but sometime organization would have to pay for service even they are not using that service.

2. Industrious password supervision plays a vital role cloud security. However, many people can access your account, so less secure it is. Anybody aware of your passwords will be able to access your information you store there.

3. Migrating data from system to cloud can pose major risks, if it not monitor and handled properly. There is need of developing migration strategy and policies that integrates well with current IT infrastructure.

\section{Solving Issues of Cloud Computing using} AWS

The AWS technique have some most useful services which are follows:-

1. Billing \& Cost Management Dashboard- This service is use to manage, track, control and monitor your monthly, month to date, or weekly expenses of using aws services. If you do not want to spend money beyond some particular amount then you can also set billing alerts in aws which gives you alert if your expenses are going beyond that particular amount.

2. Multi-Factor Authentication (MFA):-MFA is the best tool which adds extra layers of protection on top of your username and password. With MFA enabled, when user signs into aws account they will be prompted for their username and password (the first factor- what they know), as well as for an authentication code from their AWS MFA device (the second factor-what they have) taken together. These mfa increase security of your data in account.

3. AWS Migration HUB-AWS Migration Hub provides a single location to track the progress of application migrations across multiple AWS and partner solutions. Using Migration Hub, you can view the migration progress of all the resources in workloads including web and mobile applications, the application. This allows you to quickly get progress updates across all of your migrations, easily identify and troubleshoot any issues, and reduce the overall time and effort spent on your migration projects.AWS Migration Hub provides a single place to monitor migrations in any AWS region where your migration tools are available. There is no additional cost for using Migration Hub. You only pay for the cost of the individual migration tools you use, and any resources being consumed on AWS.

\section{Conclusion}

From this we can conclude that Cloud Computing has many problems like security, cost effectiveness, scalability and many more but using aws services you can overcome all this problems. Aws gives you wide variety of services which are more than 98 and many of them also have lot of variety within them. All this services can be used by very low cost even (pay per use) basis. AWS gives you flexibility which means you need not to worry about compute, processing, data storage, data security and integrity.it automatically scale up there cloud servers if data size increses.it also gives free tier access of aws console initially for a one year to get handy of using aws services. That's why aws is best among all cloud service providers in the world.

\section{Reference}

1. "Mladen A Vouk, "cloud computing", -Issues, Research and Implementation, International Conference on Information Technology Interfaces, June2008.

2. Amazon Billing and Cost Management Dashboard:

https://docs.aws.amazon.com/awsaccountbilling/la test/aboutv2/getting-viewing-bill.html

3. Multi Factor Authentication(MFA):https://docs.aws.amazon.com/IAM/latest/UserGui de/id_credentials_mfa.html

4. AWS Migration HUB:https://docs.aws.amazon.com/migrationhub/latest/ ug/getting-started.html 